livraisons

d'Histoire

de l'Architecture

\section{Livraisons de l'histoire de l'architecture}

$40 \mid 2020$

À propos des princes de Conti

\title{
Les chasses de Clemens August (1700-1761), archevêque-électeur de Cologne
}

Les chasses de Clemens August (1700-1761), archevêque-électeur de Cologne

The Hunts of Clemens August (1700-1761), Prince Bishop of Cologne

Die Jagdschlösser Clemens Augusts (1700-1761), Fürstbischof von Köln

Jean-Michel Leniaud

\section{(2) OpenEdition}

Journals

Édition électronique

URL : http://journals.openedition.org//ha/1297

DOI : 10.4000//ha.1297

ISSN : 1960-5994

Éditeur

Association Livraisons d'histoire de l'architecture - LHA

Édition imprimée

Date de publication : 3 décembre 2020

Pagination : 45-54

ISSN : $1627-4970$

Référence électronique

Jean-Michel Leniaud, « Les chasses de Clemens August (1700-1761), archevêque-électeur de

Cologne », Livraisons de l'histoire de l'architecture [En ligne], 40 | 2020, mis en ligne le 28 décembre

2020, consulté le 23 mars 2021. URL : http://journals.openedition.org//ha/1297 ; DOI : https://doi.org/ 10.4000/lha. 1297 
Par Jean-Michel LeNIAUd

\section{LES CHASSES DE CLEMENS AUGUST (1700-1761), ARCHEVÊQUE-ÉLECTEUR DE COLOGNE}

Louis-François de Bourbon (1717-1776), prince de Conti, peut être considéré à son époque, à l'instar de son cousin Louis XV, comme le plus actif veneur du royaume. Il eut un contemporain, sur la rive droite du Rhin, qui eût rivalisé avec succès : Clemens August von Bayern (1700-1761), issu de la maison de Wittelsbach, électeur du Saint-Empire, prince-évêque de Cologne. Le premier, veuf à dix-neuf ans (1736) de Louise-Diane d'Orléans, dernière fille du Régent, consacra, pour s'en consoler, deux années consécutives de son existence à la chasse, après s'être retiré dans son château de L'Isle-Adam. Puis il s'engagea dans la guerre de succession d'Autriche (1740-1748), période pendant laquelle un membre de la maison de Bavière, Charles-Albert (1697-1745), frère de Clemens August, porta sous le nom de Charles VII la couronne d'empereur des Romains de 1742 à sa mort en 1745 . Il se retira à nouveau à L'Isle-Adam, protégé par la duchesse de Châteauroux puis voué à la vindicte de la marquise de Pompadour (1757). En 1749, il obtint une charge prestigieuse qui lui donnait des ressources et une liberté considérables : celle de grand prieur de l'ordre de saint Jean de Jérusalem. En 1732, Clemens August von Bayern avait été élu grand maitre de l'ordre teutonique (Hochmeister des Deutschen Ordens) ${ }^{1}$.

\section{Clemens August von Bayern}

À la vérité, le veneur prodigue qu'était Conti, celui dont le prince de Ligne affirmait qu'il était " propre à tout et capable de rien ", ne connaissait pas l'archevêqueélecteur de Cologne. Mais outre qu'ils se trouvent à peu près de la même génération, l'un et l'autre possèdent sans doute des traits de caractère communs. Du second auprès duquel il a été placé comme représentant du roi de France, l'abbé Aunillon écrit dans ses Mémoires (p. 133) : "Il a un malheur que je lui vois commun avec presque tous les grands et les riches. Ils désirent tout et ne jouissent de rien. " En tout cas, le prince-évêque consacra beaucoup de temps et d'argent à la chasse. Son successeur, Max Friedrich de Königsberg Rothenfels (1708-1784), supprima, en raison

1. L'auteur remercie tout particulièrement le Dr Mario Kramp, directeur du Kölnisches Stadtsmuseum de Cologne, et sa collaboratrice, Ursula von Pidoll, ainsi que Christiane Winkler et Friedrich J. Becher, UNESCO-Welterbestätte, Schlösser Augustenburg und Falkenlust. Le lecteur peut aussi se reporter à Paul P. Werhan, Kurfürst Clemens August und seine Jagdschlösser, Universität für Bodenkultur Wien, Institut für Wildbiologie und Jagdwirtschaft, Departement für Integrative Biologie und Biodiversitätsforschung, Universitätslehrgang Jagdwirt/in, Vienne, janvier 2016, 68 p. 
du coût qu'ils occasionnaient, l'équipage et la fauconnerie de son budget. Moins sage, Clemens August avait chassé avec passion le héron au faucon. À Falkenlust, il s'y adonnait presque tous les soirs. Dans ses autres châteaux, il pratiquait toutes les sortes de chasse.

Clémens August avait accédé à l'âge de seize ans (1716) au siège épiscopal de Ratisbonne, puis et concomitamment, à ceux de Münster et de Paderborn. En 1723, il est désigné archevêque-électeur de Cologne, au décès de son oncle Joseph Clemens von Bayern, mais encore évêque d'Hildesheim (1724) et d'Osnabrück (1728). Proscrit par le concile de Trente, le cumul des bénéfices ne put être définitivement supprimé des états allemands par le Saint-Siège qu'après la signature du Concordat de 1801, lequel, d'ailleurs, sanctionna la suppression pour un temps du siège de Cologne.

Il était le fils de Maximilien II, électeur de Bavière et de Thérèse Cunégonde Sobieski et, outre le futur empereur Charles VII, comptait un autre frère : Jean Théodore (1703-1763) qui reprit à sa suite le siège épiscopal de Ratisbonne avant d'être désigné prince-évêque de Liège en 1744. Ce dernier était aussi un veneur acharné. Quant à Charles VII, il avait lui-même un fils, Maximilien Joseph mais ce dernier, malgré le soutien de la France, renonça à se porter candidat aux suffrages des électeurs impériaux et se rangea sous la bannière des Habsbourg, la maison rivale. Il en résulta l'élection de l'époux de Marie-Thérèse, Joseph Étienne de Lorraine. Pendant ce temps, Clemens August recevait 600000 livres par an de la France, qui achetait la fidélité, au demeurant douteuse, d'un prince-évêque que gratifiaient non moins les ennemis du Royaume, les Provinces-Maritimes, GrandeBretagne et Pays-Bas. Avec ces significatives ressources, le prince-évêque faisait construire des châteaux de chasse.

\section{Aunillon : un abbé diplomate}

Pour se garantir la possible loyauté de Clemens August, la cour de France avait dépêché un ecclésiastique comme chargé d'affaires à celles du prince-évêque, dans l'idée sans doute que leur état commun garantirait des relations confiantes. Il en fut ainsi : tout le temps de sa mission (1744-1747), l'abbé Aunillon occupa la place de confident de l'Électeur, lui fit écarter ceux de ses conseillers qui recevaient des émoluments des Provinces-Maritimes et fut vivement regretté de Clemens August lorsque Versailles rappela son représentant.

L'abbé Aunillon (1688-1760) était, de fait, un "personnage». On le connaît par ses mémoires qui parurent de façon posthume en deux volumes en 1808 sous le titre: Mémoires de la vie galante, politique et littéraire de l'abbé Aunillon Delaunay du Gué, ambassadeur de Louis XV près le prince électeur de Cologne. Sa carrière avait commencé par les charges de grand vicaire de l'archevêque de Vienne, puis de l'évêque d'Évreux (1714-1733). Mais il avait préféré les plaisirs de la vie parisienne, la passion des spectacles, la compagnie des jeunes actrices et les soirées en ville. 
L'une de ses pièces, La Comédie des amants déguisés, inspira Marivaux dans le Jeu de l'amour et du hasard. En 1742, il se trouvait proche de Voltaire. On aurait pu penser qu'il fréquentait les salons du prince de Conti. On lit tout de même (t. I, p. 292-293) : "J'ai acquis [...] dans les voyages fréquents que je fis à leur maison de Pontoise, la confiance et j'ose dire l'amitié du prince de Conti et du comte de Clermont [Louis de Bourbon Condé, 1709-1771]. Je jouis peu des bontés du premier. La mort l'enleva à la fleur de l'âge. " Mais vu les dates, c'est du père de LouisFrançois qu'il s'agit, Louis-Armand (1696-1727), celui que ses contemporains désignaient sous le sobriquet de "Singe vert" et qui mourut prématurément de la petite vérole. Quant à la proximité d'Aunillon avec le comte de Clermont, on y vit la preuve de son appartenance à la franc-maçonnerie ${ }^{2}$.

Le deuxième volume des mémoires raconte à la Quatorzième lettre, Mémoires de ma légation auprès de l'Électeur de Cologne en 1744 jusqu'à la fin de 1747, les circonstances de son séjour à la cour du prince-évêque à Bonn et autres lieux. Il avait été nommé sur proposition de d'Argenson, alors secrétaire d'État des Affaires étrangères. Aunillon en dit que ce dernier l'« estimait non comme un saint mais comme un homme aimable" et ajoute qu'il avait été choisi par lui au poste de Bonn parce qu'informé par une indiscrétion que le portefeuille lui avait été confié, il avait été le premier à l'en féliciter. Sur place, il s'employa avec beaucoup de sérieux à ranger le prince-évêque parmi les amis de la France alors que ce dernier avait conclu un traité avec les Puissance maritimes, recevait de gros subsides de leur part et leur donnait en échange 10000 hommes d'arme. Il avait fort à faire sur ce point car, de naturel irrésolu, le prince-évêque témoignait d'une loyauté à tout le moins fragile. Pourtant, écrit le chargé d'affaires (t. II, p. 136), il donnait la preuve de "plus d'esprit que sa condition n'en comportait".

Le prince-évêque entrait-il dans la catégorie des éternels insatisfaits? C’est ce que pense l'abbé Aunillon : "Ils n'[ont] d'autres [plaisirs...] que d'être toujours en quête pour le trouver et d'aller sans cesse le chercher où ils ne le trouveront pas. " (t. II, p. 133). Il fallait donc suivre pas à pas le prince-évêque pour lui éviter d'être placé sous la coupe de quelque influence extérieure. Aunillon partageait avec Clemens August le goût de l'architecture et des jardins et en retirait la confiance du prince $^{3}$. Il l'accompagnait très fréquemment, c'est-à-dire presque tous les soirs, dans sa résidence favorite de Falkenlust ; il était son hôte dans son palais, près de Brühl, à Augustusburg. Et surtout, il faisait tout pour l'accompagner d'un lieu à l'autre dans ses déplacements pour chasser le héron au faucon. Telle était la chasse favorite du prince. C'est en tenue de veneur, un faucon à son poignet gauche, qu'il s'est fait portraiturer en pied, par Peter Jakob Horemans (1700-1776) ${ }^{4}$.

2. Pierre Chevallier, Les Ducs sous l'acacia. Les premiers pas de la Franc-Maçonnerie, 1725-1743, Paris, Vrain éd., 1994, 232 p., p. 91.

3. Mémoires, op. cit., p. 97 : "Quelque goût pour les bâtiments et pour les jardins m'attira la confiance d'un prince qui en fait une de ses principales occupations, au point qu'il ne faisait plus rien sans me consulter."

4. Ce tableau est conservé à Falkenlust. 


\section{Les châteaux de chasse du prince-évêque}

On a pu montrer que les contacts assidus que la cour de France entretenait avec celle du prince électeur ne concernaient pas seulement les affaires diplomatiques mais les échanges artistiques. Plus exactement, ils ont permis à Clemens August de se tenir au plus près de la création et de la mode à Versailles et à Paris. Selon une heureuse formule de Martin Miersch, les diplomates français ont rempli un rôle significatif dans l'apprentissage du "bon goût " par un membre d'une maison, les Wittelsbach, déjà liée et depuis longtemps il est vrai, avec la famille royale ${ }^{5}$. Clemens August, pour sa part, s'était rendu en France en 1724 pour le mariage de Louis XV à Fontainebleau mais il n'y revint pas : après 1745 , le renoncement des Wittelsbach à la couronne impériale atténuait, il est vrai, l'intérêt du Royaume pour le rôle diplomatique du prince-électeur. Ce qui ne l'empêcha pas de poursuivre son œuvre artistique autant en architecture et en arts décoratifs qu'en musique. Une publication relativement récente a fait le point là-dessus, sous la direction de Frank Günter Zender: Eine Gesellschaft zwischen Tradition und Wandel. Alltag und Umwelt im Rheinland des 18. Jahrhunderts ${ }^{6}$. On y retiendra notamment l'étude sur la chasse de Rolf Hocker et de Wolfgang Wessel sur la vènerie du prince : "Die Jagd in Kurköln zur Zeit von Clemens August ${ }^{7}$ " et celle, sur la musique, de Claudia Valder-Knechtges, "Die kurfürstliche Hofmusik im 18. Jahrhundert ${ }^{8}$ ». Les deux sujets sont, au reste, liés : on sait, par exemple, que Johann Ries (1723-1784), trompettiste chez l'Électeur, a composé pour lui une messe de saint Hubert avec Jagdhörner ${ }^{9}$ en 1756.

Clemens August ne posséda pas moins de six châteaux, dont cinq châteaux de chasse. Le premier, Augustusburg, se situe aux environs de Brühl et a été inscrit sur la liste du patrimoine mondial en 1984. Sa construction a commencé en 1725 sous la direction de l'architecte Johann Conrad Schlaun (1695-1773) à l'emplacement d'une ancienne forteresse. Elle été poursuive par François de Cuvilliés l'Ancien (1695-1768) pour les façades et l'intérieur. L'escalier en a été réalisé par Johann Balthasar Neumann (1743-1748).

5. Voir Martin Miersch, Das Bild des Electeur Soleil: Herrscherikonographie des Rokoko am Beispiel des Kölner Kurfüsten und Deutschordenshochmeisters Clemens August (1700-1761), Marburg, N. G. Elwert, 2007.

6. Cologne (Dumont), 1999.

7. P. 227-246.

8. P. 151-17.

9. Je préfere laisser le terme allemand car on n'est pas certain qu'il s'agisse à proprement parler de trompes de chasse ou de cors par force qui ne sont pas tout à fait identiques. Le manuscrit de cette messe se trouve à la bibliothèque de Modène et a été édité par Inge Forst en 1989. Cette musicologue a publié un article sur cette messe dans Kirchenmusikalisches Jahrbuch, t. 7, p. 35-40, renseignements fournis très amicalement par Catherine Massip. L’Accademia di San Huberto a fait créer cette œuvre en avril 2018 mais l'a attribué, chauvinisme italien (?), à Pietro Torri en la datant de 1722, ce qui est stylistiquement impossible. Johann Ries était le grand père du compositeur et pianiste Ferdinand Ries. 


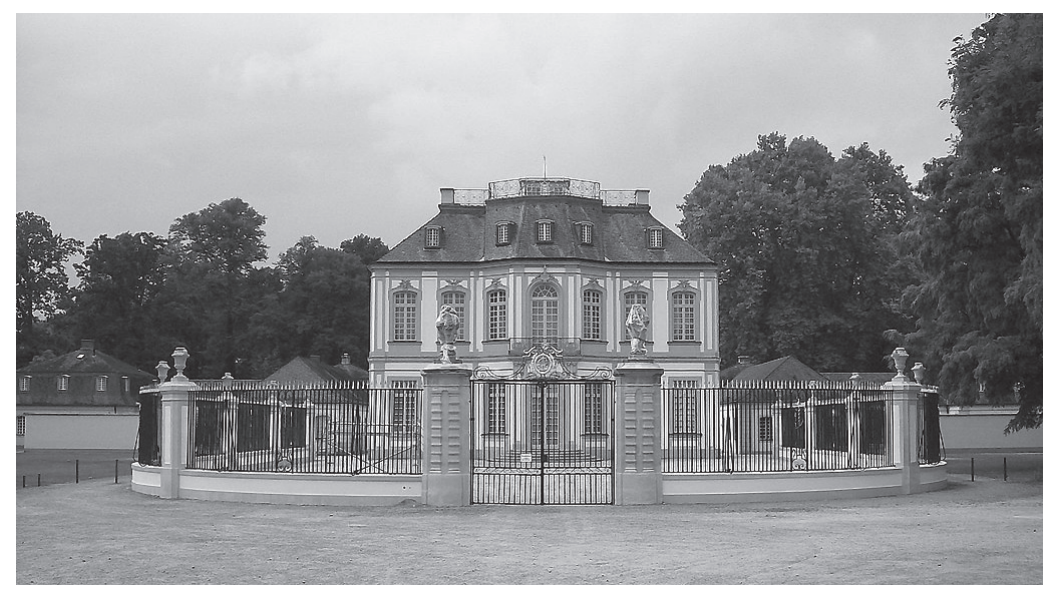

Ill. 1 : Falkenlust, façade principale. (C) Stefan Didam. Schmallenberg / CC BY-SA.

\section{Falkenlust}

À côté d'Augustusburg et inscrit la même année 1984 sur la liste du patrimoine mondial se trouve la résidence que le prince-évêque avait destinée à la chasse au faucon : Falkenlust i.e. : désir de faucon ${ }^{10}$. Conçu par François de Cuvilliés l'Ancien, il fut construit à partir de 1725. Mozart qui se produisit en 1766 au Temple dans le salon du prince de Conti était passé à Falkenlust en 1763 mais le très mélomane prince-évêque était décédé depuis trois ans. Un corps de bâtiment de sept travées, sur deux niveaux couverts d'un comble pentu, ainsi qu'un avant-corps central à trois pans coupés, marque la partie centrale de la façade principale (ill. 1). De l'autre côté, le centre de la façade se signale de même par une double paire de colonnes (ill. 2). Les communs forment une harmonieuse composition de part et d'autre : on y trouvait les écuries, la fauconnerie et probablement un chenil si on admet qu'il était impossible que le prince-évêque n'en possédât pas.

Du pavillon de chasse partait une grande avenue de part et d'autre de laquelle s'étendait un parc forestier dans lequel des allées étaient tracées de façon à ménager des convergences qui fournissaient des points de vue panoptiques pour observer les déplacements du gibier chassé depuis la terrasse supérieure.

L'iconographie des décors intérieurs est largement consacrée à la chasse, au héron et au faucon, en particulier celle des carreaux de céramique en camaïeu bleu à l'escalier qu'avait conçu Stephan Laurenz de la Roque (1695-1742) vers 1730.

\section{Clemenswerth}

Le pavillon de chasse de Clemenswerth (i.e. : La valeur de Clément) a été construit de 1737 à 1747 dans l'Emsland, près de Sögel en Basse-Saxe. De même qu'Augustusburg, il a été conçu par Johann Conrad Schlaun. De plan cruciforme,

10. Wilfried Hansmann, Schloss Falkenlust in Brühl, Worms, Wernersche Verlagsgesellschaft, 2002, $160 \mathrm{p}$. 


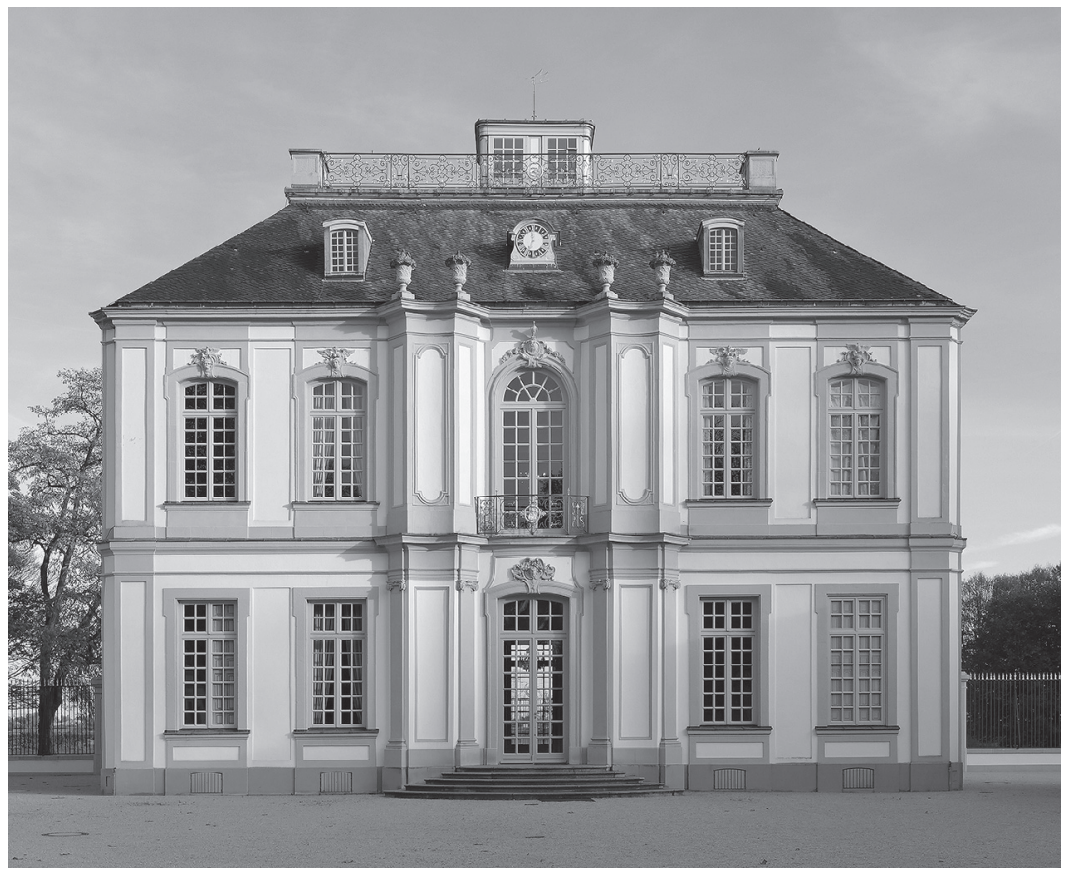

Ill. 2 : Falkenlust, façade arrière. (C) Wikipedia. Creative commons BY-SA.

il est disposé au centre d'une ample composition convergente, dont chacun des rayons est marqué d'un pavillon plus petit. Ce mode de composition du paysage destiné à ménager des points de vue sur la nature, sur le gibier et sur la chasse anticipe sur des compositions similaires tel le pavillon de la Muette que Louis XV commanda à Ange Jacques Gabriel en forêt de Saint-Germain à l'extrême fin de sa $v^{1}{ }^{11}$. Une exposition organisée en 1987 a présenté ${ }^{12}$ un Parforcehorn de $527 \mathrm{~cm}$ de long, c'est-à-dire plus long que la trompe à la Dampierre $(454,50 \mathrm{~cm})$, et de $62,50 \mathrm{~cm}$ de diamètre (contre $72 \mathrm{~cm}$ pour la Dampierre). L'instrument sonne en si, c'est-à-dire une tierce mineure plus bas que la Dampierre, en ré. L'instrument réalisé en 1722 par Johann Paulus Frank à Hildburghausen est conservé au Germanisches Nationalmuseum de Nuremberg. Mais, à vrai dire, rien n'indique dans la notice du catalogue que cet instrument provienne du château de Clemenswerth ou des chasses de Clemens August.

11. Voir là-dessus Eckard Wagner, "Schloss Clemenswerth. Ein Höhepunkt jagdlicher Zentralanlagen in Europa ", Clemens August Fürstbischof, Jagdherr, Mäzen. Eine kulturhistorische Ausstellung aus Anlass des 250 jährigen Jubiläums von Schloss Clemenswerth, hrsg. vom Landkreis Emsland Meppel/ Sögel, Bramsche, Rasch Verlag, 1987, 504 p., p. 119-148 et plus particulièrement p. 122-132. On a reconnu comme modèles de Clemenswerth, la pagode de Radstadt, l'ermitage de Waghäusel et le château de Marly-le-Roi.

12. P. 499. 


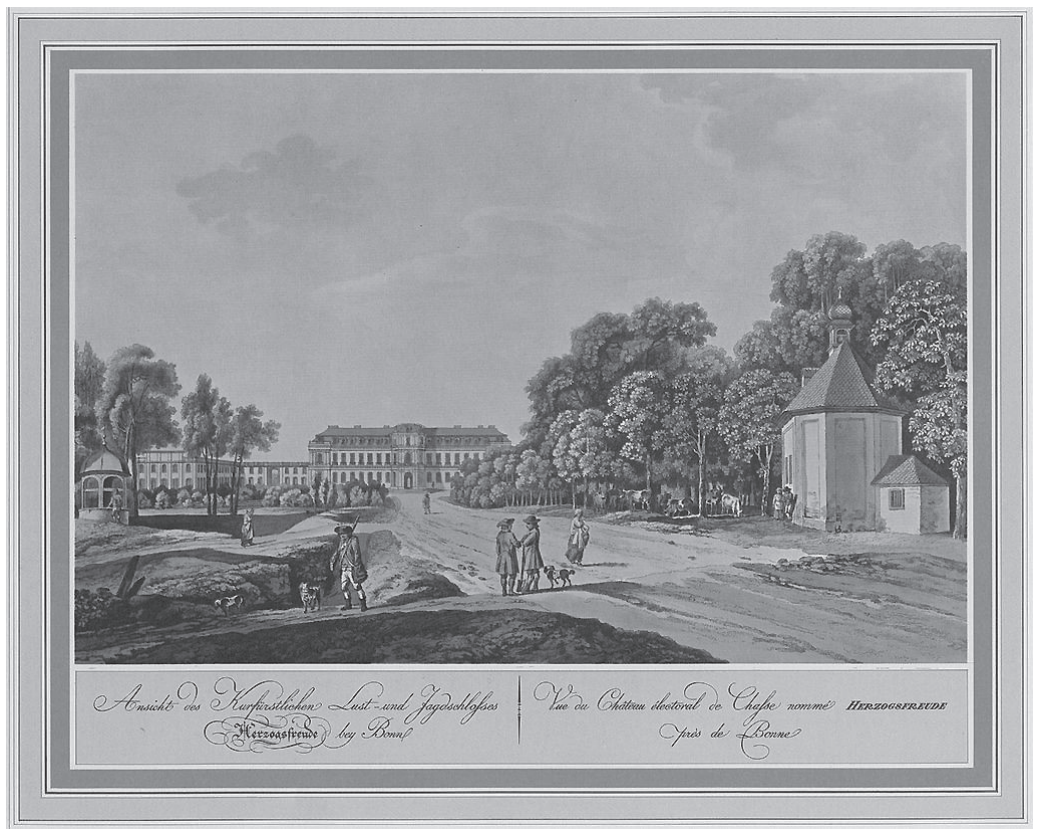

Ill. 3 : Herzogsfreude, façade principale, 1753-1755, Johann Ziegler del. (C) Wikipedia commons. Libre de droits.

\section{Herzogsfreude}

Clemens August possédait un troisième château de chasse, Herzogsfreude (i.e. : le plaisir du duc) à côté de Bonn, à Röttgen. Construit entre 1753 et 1755 pour la chasse à courre, il demeura sans utilisation après son décès, puis fut nationalisé et mis aux enchères en 1804 à l'instigation de l'occupant français (ill. 3). Ce que l'affiche de la vente désignait comme "les restes du château de Röttgen " en raison du mauvais état de la construction fut acheté par le Dachdecker (i.e. : terrassier) Peter Lander pour la modique somme de 3550 francs. Réduit au triste sort de carrière de matériaux de récupération, il fut dépecé au cours des années suivantes.

On connaît l'organisation de l'édifice grâce, entre autres, à une représentation de Johann Ziegler (1749-1812), gravure en couleur datée de 1798 d'après un dessin de Johann Janscha ${ }^{13}$. Constitué d'un corps principal avec avant-corps central à pans coupés et de deux ailes, il formait le cour d'un dispositif en étoile selon le principe qui avait gouverné la construction de Falkenlust, avec de larges allées tracées dans un vaste parc: toutes étaient alignées par rapport au palais électoral de Bonn et aux châteaux de Brühl (Augustusburg et Falkenlust), eux-mêmes distants de vingt-cinq km environ. Préalablement à la construction du château, le Kottenforst, massif forestier à proximité de Bonn, avait été aménagé pour la chasse. En 1750, le prince-évêque y fit construire le Schönwaldhaus, un bâtiment du service forestier.

13. Osterreichschisches Biblionationalbibliothek Wien. 


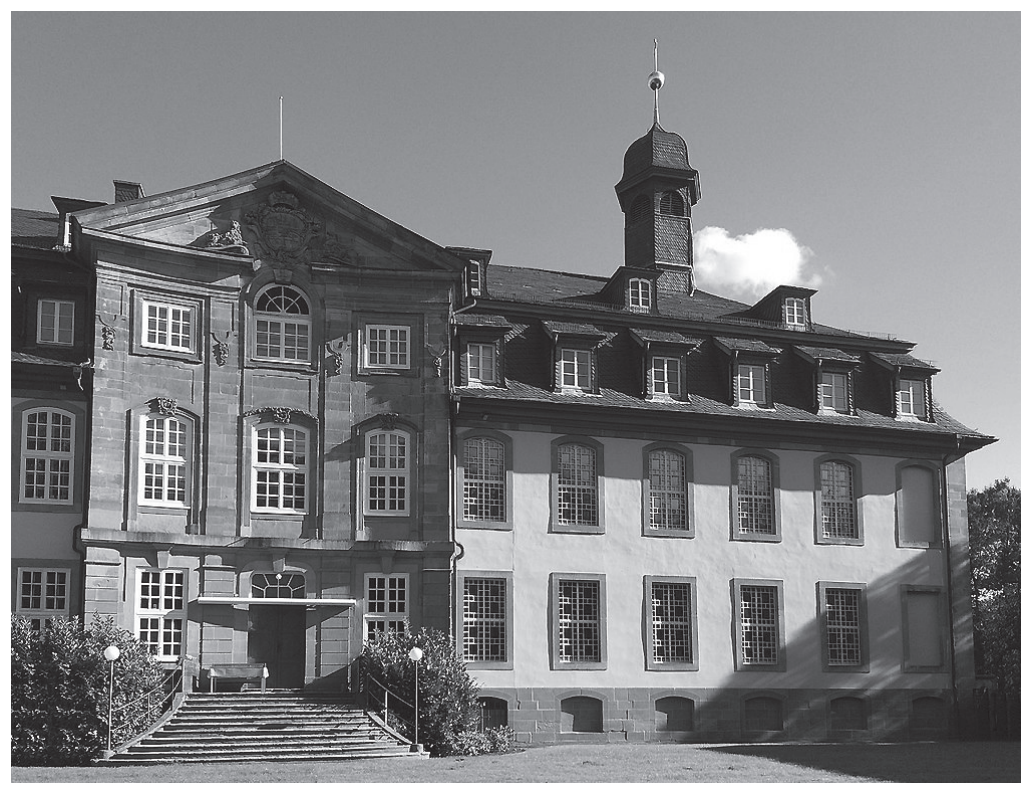

Ill. 4 : Liebenburg, élévation extérieure. () Wikipedia. Creative Commons CCO 1.0.

Il avait, entre temps, érigé une chapelle sous la double invocation de saint Venantius, guérisseur de la lèpre et de saint Hubert, patron des chasseurs et guérisseur de la rage et y installa en 1746 un ordre de chevalerie composé de douze membres recrutés dans des familles de haute noblesse sous la devise de "Aussi clément qu'auguste ", par référence aux prénoms qu'il portait : " hochadelige Ritterorden von der Gütigkeit, ou Ordre de la Clémence ». Le secrétariat de l'ordre était assuré par le prieur du Kloster auf dem Kreuzberg, prieuré franciscain installé à Bischofsheim an der Rhön.

\section{Schloss Liebenburg}

Un autre château se trouvait à côté de Goslar dans le diocèse d'Hildesheim dont Clemens August était l'évêque, le Schloss Liebenburg (i.e. : château des amours). À l'emplacement des restes d'un château médiéval qu'il avait fait détruire, Clemens August fit ériger une nouvelle construction par l'architecte de Bonn, Michael Leveilly (1694-1762), d'après les plans de François de Cuvilliés l'Ancien (ill. 4). Le chantier commença en 1754 mais la guerre de Sept ans et le manque de ressources empêchèrent l'achèvement du chantier.

\section{Jagdschloss Entenfang}

Un dernier château, plus modeste par son architecture que les précédents, se trouve à Wesseling, non loin de Brühl. Le Jagdschloss Entenfang (i.e. : château de chasse aux canards) avait été édifié avant 1735, c'est-à-dire dix ans après Falkenlust 


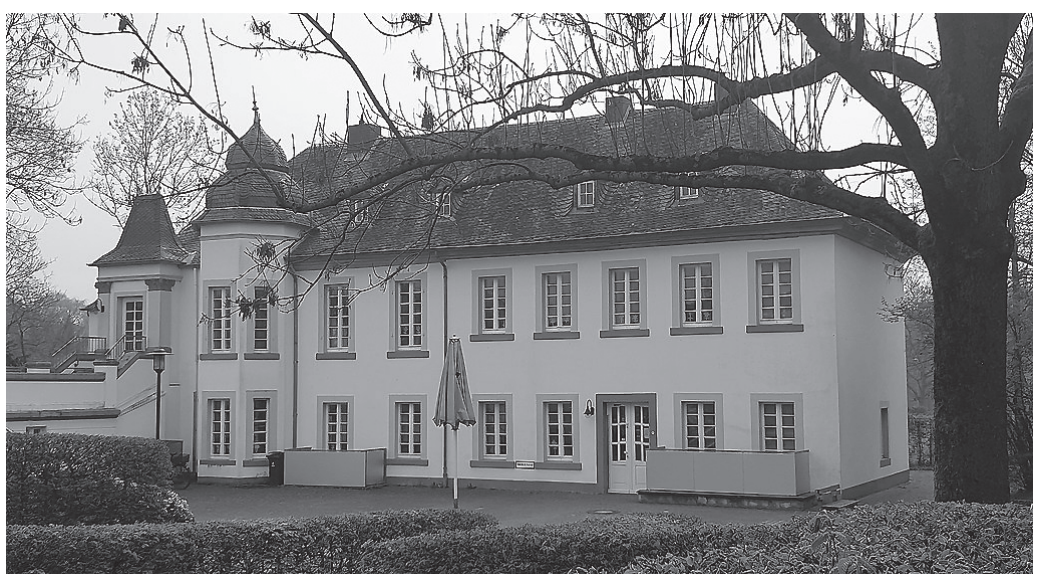

Ill. 5 : Entenfang, élévation extérieure. (C) Wikipedia. Creative Commons CCO 4.0.

(ill. 5). Le bâtiment s'élève sur un plan rectangulaire et une hauteur de deux niveaux, eux-mêmes surmontés d'un haut comble percé de lucarnes. Ce qui semble le reste d'une ancienne tour interpénètre le corps central.

Les cinq châteaux de chasse du prince-électeur de Cologne l'emportent de façon évidente sur les propriétés du prince du sang, S.A.S. Louis-Francois de Bourbon, prince de Conti, à Issy-lès-Moulineaux et à l'Isle-Adam. Il est vrai que ces dernières ont presque toutes disparu (L'Isle-Adam, Issy-lès-Moulineaux, à l'exception de Trie et de Stors), alors que deux constructions de Clemens August sont inscrites aujourd'hui sur la liste du patrimoine mondial. Il est vrai aussi que le turbulent seigneur de L'Isle-Adam possédait un équipement de vènerie si considérable et prestigieux que seul, Louis XVI, par le truchement de son frère, le comte de Provence, put le racheter, alors que les équipages du prince-évêque n’ont laissé de souvenir ni par les meutes ni par la cavalerie qui les constituaient. Mais à s'en tenir à la seule architecture, les châteaux de Clemens August de Bavière trouvent leurs seuls rivaux, si on écarte ceux des rois Bourbons, dans les entreprises des ducs de Saxe à Moritzburg et celles de la dynastie de Savoie : la palazzina di caccia de Stupinigi et le château royal de Venaria reale, l'un et l'autre à côté de Turin ${ }^{14}$.

Jean-Michel LENIAUD Directeur d'études à l'École pratique des hautes études (équipe Histara)

14. Là-dessus voir Andrea Merlotti, Le Cacce Reali nell'Europa dei principi, Centro studi della reggia di Veneria. La civiltà delle corti, Leo S. Olschi editore, 2017, 350 p. 
